\title{
Neuf familles de termes médicaux du Moyen Âge : une analyse morphologique
}

\author{
Céline Szecel \\ KU Leuven \\ Celine.Szecel@kuleuven.be
}

Résumé. L'objectif de cet article est de répondre à une question de recherche concernant l'analyse morphologique des néologismes médicaux du Moyen Âge: pourquoi certains néologismes français créés dans le domaine médical à l'époque médiévale parviennent-ils à se maintenir jusqu'en français moderne, alors que d'autres disparaissent après un certain temps ? Notre hypothèse est que la morphologie, et la transparence constructionnelle, en particulier, joue un rôle crucial dans la préservation de ce lexique. Concrètement, des termes qui présentent une relation formelle proche de l'élément latin dont ils sont issus se maintiendraient mieux que des créations françaises originales, c'est-à-dire des dérivations ou des compositions réalisées à partir de bases morphologiques françaises. Par ailleurs, les familles de lexèmes bien établies comprenant des corrélations systématiques et transparentes entre les formes et leur signification favoriseraient le renforcement et le maintien d'une terminologie. L'étude de neuf familles morphologiques montre que ces deux critères semblent être importants pour la survie des néologismes. Néanmoins, la taille de la famille morphologique ne paraît pas influencer la survie du néologisme. Dans la dernière étape de notre analyse, nous illustrons comment les propriétés sémantiques et formelles des dérivations affixales peuvent être représentées à l'aide de la Morphologie des Constructions (Booij, 2010).

\footnotetext{
Abstract. Nine families of medical terms in the Middle Ages: a morphological analysis. The objective of this article is to answer the following research question concerning the morphological analysis of medieval medical neologisms: why do certain French neologisms that emerged in the field of medicine during the Middle Ages manage to survive, while others disappear after some time? Our hypothesis is that morphology, in particular constructional transparency, contributes in a crucial manner to lexical preservation: more specifically, words showing a close formal relation with the Latin equivalent from which they were borrowed, should stand the test of time better than original French creations. Additionally, being embedded in large word families with systematic and transparent correlations between form and meaning should also reinforce the use of these neologisms and help them survive. The study of nine morphological families shows that both criteria seem to be important for the survival of these neologisms. However, the size of the word family does not seem to have an influence on its survival. In a last stage of our analysis, we illustrate how Construction Morphology (Booij,
} 
2010) can be used to represent the semantic and formal properties of affixal derivations.

\section{Introduction}

Cet article a pour objectif de répondre à une question de recherche concernant l'analyse de néologismes médicaux du Moyen Âge. Ainsi, nous examinons les raisons de la survie en français moderne de certains néologismes médicaux créés au cours de cette période, comme par exemple fistule «fistule », et de la disparition d'autres, comme festre «fistule » et afistuler « former une fistule ».

Les néologismes analysés appartiennent au domaine médical de la pathologie médiévale et ont été relevés dans un corpus numérisé, CHrOMed (1.665.663 mots) et lemmatisé de textes médicaux du $13^{\mathrm{e}}$ au $15^{\mathrm{e}}$ siècle, constitué surtout de traductions de textes sources latins, mais aussi de quelques textes immédiatement composés en français. Au total, 270 néologismes ont été retenus, désignant surtout des maladies, aussi bien physiques que mentales ${ }^{1}$, mais aussi des pustules, abcès ou tumeurs.

Précisons par ailleurs que nous retenons comme néologiques non seulement les termes médicaux attestés pour la première fois dans le corpus, mais aussi ceux dont les premières attestations se trouvent dans d'autres textes du Moyen Âge. Leur caractère néologique a été vérifié à l'aide des dictionnaires les plus importants ${ }^{2}$ pour le français, afin de distinguer les emprunts et les créations françaises, c'est-à-dire les dérivés ou composés, en adoptant la typologie toujours valable établie par Deroy (1956 : 215-234). Les formes héréditaires, en revanche, ne sont pas considérées comme des néologismes. Vu l'origine latine de la langue française, ces formes ont un étymon latin, mais ont subi des changements phonétiques au cours de leur évolution du latin au français, comme par exemple festre qui provient du latin fistula «fistule». Nous n'étudions pas non plus les xénismes, c'est-à-dire les termes caractérisés par une forme étrangère et présentés comme relevant d'une autre langue, ni les lexèmes dont la forme complète est étrangère, mais dont l'appartenance à une autre langue n'a pas été précisée (Deroy, 1956 : 224). Pour les besoins de cette contribution, nous avons fait abstraction des variantes ${ }^{3}$ du terme présentes dans notre corpus.

La survie d'un néologisme implique qu'il se lexicalise, c'est-à-dire qu'il est accepté par le locuteur comme un terme autonome qui fait partie du vocabulaire de la langue, se différencie des autres mots grâce à son caractère unique et possède un sens. Dans ce cas, le néologisme s'intègre dans le lexique du locuteur et perd son statut de mot nouveau. Toutefois, certains néologismes ne parviennent pas à se lexicaliser et disparaissent de la langue, parfois même après avoir existé pendant plusieurs siècles (Goyens \& Van Tricht, $2015: 390)$.

Dans notre contribution, nous décrivons tout d'abord les critères pouvant influencer la survie ou la disparition des néologismes médicaux du Moyen Âge, suivis de notre hypothèse de recherche (1). Dans la deuxième partie, nous présentons la terminologie utilisée ainsi que le cadre théorique, à savoir la Morphologie des Constructions, avant d'analyser neuf familles morphologiques figurant dans notre corpus (2). Les résultats de cette analyse sont repris dans la troisième section de cette contribution afin de répondre à notre question de recherche (3), pour terminer par la réanalyse de ces résultats selon les principes de la Morphologie des Constructions (4).

\section{Critères et hypothèse de recherche}

Le maintien ou la disparition des néologismes peuvent être provoqués par une multitude de facteurs (Goyens \& Van Tricht, 2015 : 390-391). Ainsi, il est évident qu'un terme n'est pas maintenu si la réalité qu'il désignait disparaît. Concrètement, de nouvelles découvertes 
médicales peuvent mener à l'abandon d'un terme. Peut-être est-ce pour cette raison que cacochie ("gonflement des membres provoqué par de mauvaises humeurs »), concept issu de la théorie humorale médiévale, a disparu de la langue. Les termes cacochime (« (patient) atteint de cacochie, plein de mauvaises humeurs») et cacochimie («corruption des humeurs, pouvant entraîner la cacochie »), quant à eux, ont connu un changement sémantique, probablement pour le même motif, et ont reçu une signification plus figurée. ${ }^{4}$ Par ailleurs, il faut également tenir compte du contexte socio-historique dans lequel les textes de notre corpus ont vu le jour, et donc aussi les néologismes analysés. Au Moyen Âge, les textes scientifiques étaient presque exclusivement composés en arabe, grec ou latin (Lusignan, 1989). À cette époque, les clercs ou les traducteurs français maîtrisaient parfaitement le latin, cette langue devenant même leur première langue dans un contexte scientifique (Van Tricht, 2015 : 561-562). Cependant, au 13 ${ }^{\mathrm{e}}$, mais surtout au 14 ${ }^{\mathrm{e}}$ siècle, les savants français commencèrent à traduire les textes scientifiques et médicaux latins en langue vernaculaire. Néanmoins, le latin continua à être la langue de référence médicale tout au long de la Renaissance et même des siècles suivant cette période, avant que les langues vulgaires, et en particulier le français et l'anglais, ne deviennent dominantes dans le contexte médical (Wermuth \& Verplaetse, Forth.). Dès lors, le prestige du latin en tant que langue de la médecine pourrait influencer la survie des néologismes et favoriser ceux d'origine latine (Goyens, 2013 : 54).

En outre, Goyens et Van Tricht $(2015$ : 390, 396) ont montré, grâce à une analyse menée sur un corpus restreint de quatre textes médicaux du Moyen Âge, que la popularité d'une œuvre médiévale ainsi que l'autorité et le prestige de son auteur peuvent aider un néologisme à se maintenir: "plus une œuvre a de lecteurs ou d'auditeurs, plus sa terminologie a des chances de s'imposer ». Afin de s'assurer de la bonne compréhension d'un néologisme par leurs lecteurs, les traducteurs médiévaux dotaient souvent les néologismes de gloses explicatives. Il est donc intéressant d'examiner la présence ou l'absence de ces gloses auprès des néologismes au sein des textes médicaux qui les introduisent. Il serait également opportun de vérifier si un auteur ou traducteur est systématique dans l'emploi d'un néologisme au sein d'un même texte. Par ailleurs, si le néologisme est employé dans d'autres textes et que sa fréquence est élevée, cela augmenterait ses chances de survie (Goyens \& Van Tricht, 2015 : 390, 394-396).

Toutefois, ces critères ne suffisent pas à eux seuls pour expliquer le maintien ou la disparition d'un néologisme médical du Moyen Âge, les études de Ducos (1998 : 246-247) et de Städtler (2007 : 50-51) semblant impliquer que les créations françaises auraient plus de difficultés à se maintenir que les néologismes provenant du latin. Goyens (2013 : 46, qui se base sur Goyens \& Van Tricht, 2015: 393) a en effet montré que la majorité des néologismes médicaux créés au cours du Moyen Âge sont des emprunts au latin $(77 \%$, soit 96 des 125 néologismes médicaux), alors que seulement 23\% des néologismes sont des créations françaises. Ces résultats ne sont pas surprenants, vu la situation sociolinguistique du Moyen Âge, le latin étant la langue par excellence de la médecine médiévale (Lusignan, 1989). En outre, les morphèmes lexicaux, c'est-à-dire les bases du latin sont aisément reconnaissables dans les dérivés et composés qu'ils forment, rendant donc le latin transparent sur le plan de la morphologie lexicale, à l'inverse du français (Goyens, 2013 : 52-53).

Des facteurs morphologiques pourraient dès lors influencer la survie ou la disparition des néologismes médicaux du Moyen Âge. Pour définir ces critères, Goyens (2013 : 49-50) se base sur les résultats des travaux dans le domaine de la psycholinguistique, comme par exemple ceux publiés par Dal (2003), tels Meunier (2003 : 26-31) et Namer (2003), qui ont conduit des expériences concernant la création de néologismes, et en particulier, la dérivation en français moderne. Pour qu'un mot nouveau soit accepté par un locuteur, l'affixe doit être aisément reconnaissable et le terme concerné doit faire partie d'une famille de mots où des liens morphologiques se tissent entre les membres de cette famille. Il faut 
aussi que la taille de cette famille soit importante, que la transparence sémantique permette de distinguer la racine et l'affixe, et, enfin, que le nombre de syllabes du terme ne soit pas trop élevé. Même s'il s'agit de résultats d'expérimentations pour le français moderne, il est possible de les appliquer au français médiéval, le traducteur médiéval et son public disposant d'un lexique mental, tout comme le locuteur moderne. Ce lexique mental est défini par Meunier (2003: 24) comme « un système organisé de connaissances susceptibles de permettre à l'utilisateur un accès rapide et efficace aux informations lexicales qui concernent des dizaines de milliers de mots ", ces dernières pouvant aussi être d'ordre morphologique. $\mathrm{Vu}$ le bilinguisme du traducteur médiéval, celui-ci possède probablement au moins deux lexiques mentaux, à savoir celui du français et du latin, qu'il peut exploiter lors de la création de néologismes.

Si nous continuons nos réflexions à propos des néologismes cacochie, cacochime et cacochimie, l'on pourrait se demander pourquoi ces deux derniers termes parviennent à se maintenir formellement, avec un changement de sens, alors que cacochie disparaît complètement. ${ }^{5} \mathrm{Si}$ la disparition de la réalité médicale suffisait à expliquer la disparition des néologismes concernés, cacochime et cacochimie n'auraient pas pu survivre. Des critères morphologiques pourraient-ils expliquer ce phénomène ?

$\mathrm{Vu}$ les limites imparties par cette contribution, nous avons sélectionné trois facteurs morphologiques et formulé l'hypothèse de recherche suivante: la survie de ces néologismes médicaux dépend de leur relation formelle avec l'élément latin dont ils sont issus. Plus cette relation est transparente et plus le néologisme appartient à une famille morphologique bien établie, mieux le néologisme se maintiendra, alors que les créations françaises indigènes disparaîtront plus facilement, en tout cas dans un emploi médical. Nous voudrions par ailleurs vérifier si les familles de lexèmes manifestant des corrélations systématiques et transparentes entre les formes et leur signification aident une terminologie à se renforcer et à se maintenir. ${ }^{6}$

\section{Analyse de neuf familles morphologiques}

\subsection{Terminologie et cadre théorique}

Avant de vérifier notre hypothèse en analysant plusieurs familles morphologiques, il est nécessaire de définir les notions de famille morphologique, famille étymologique, racine et base. $^{7}$ Nous adoptons le concept de famille morphologique tel qu'il est entendu par Hathout (2011 : 262), pour qui « les familles dérivationnelles morphologiques, ou plus simplement familles morphologiques, correspondent à des relations morphologiques intermédiaires qui s'établissent entre des lexèmes dont les formes partagent leurs propriétés les plus spécifiques ». Les termes appartenant à une même famille morphologique ont donc des propriétés sémantiques et formelles en commun, comme par exemple dérivation, dériveur, dérivée, dérive, dérivationnel, dérivationnellement, dérivable, dérivatif et dériver (Hathout, 2011 : 262). La racine, appelée radical par Hathout (2011 : 264), est l'élément formel partagé par tous les membres de la famille morphologique, qui permet de reconnaître leur adhérence à cette famille. La base, quant à elle, est la partie de la forme qui subsiste lorsque l'on enlève les affixes (Apothéloz, 2002: 15). Sudat- serait donc la base du terme desudation " pustule provoquée par la sueur », qui est attesté dans notre corpus et qui fait partie des termes pathologiques que nous analysons, et sud-, sa racine. Ensuite, nous avons cherché/identifié dans notre corpus les autres mots construits à partir de cette racine sud- et nous en avons relevé trois, à savoir sudoral, sudorable et resudation. La même méthode a été appliquée afin d'identifier les autres familles morphologiques analysées dans cette contribution et dont les membres ont tous un sens exclusivement médical, à l'inverse d'autres familles présentes dans notre corpus. Parfois, deux familles morphologiques 
différentes remontent à une même racine latine et forment, dès lors, une famille étymologique. Ainsi, la famille morphologique de sudoral, sudorable, resudation, desudation, surtout composée de formes empruntées au latin, et celle des termes héréditaires suer, sueur constituent une famille étymologique, parce qu'elles proviennent toutes deux de la racine sud-.

Concrètement, nous avons analysé les neuf familles morphologiques suivantes, dont les termes ont été relevés dans notre corpus ${ }^{8}:$ (1) cacochie, cacochime, cacochimie, cacochimeux, cacochimique; (2) chancre, chancreux, enchancrir, achancrir ; (3) flegme, flegmatique, flegmasie, leucoflemantie; (4) dissinterie, dissinterique; (5) apoplexie, apoplectique ; (6) apostume, apostumation, apostumeux, apostumer ; (7) asme, asmatique ; (8) sudoral, sudorable, resudation, desudation ; et enfin, (9) suer, sueur. ${ }^{9}$

Pour analyser ces lexèmes, nous les avons décomposés en base/racine et affixes, mais nous avons rencontré des difficultés quant au statut de certains éléments. Dans notre corpus, nous avons par exemple relevé le terme desudation, dont l'élément -at- reste difficile à traiter. Cet élément fait-il partie intégrante de la base ou plutôt du suffixe ? Doit-on en outre considérer -tion comme le suffixe, comme le propose le TLFi, ou plutôt -ion, comme le suggère Amiot (2011: 24) ? Ni le TLFi, ni Amiot (2011) n'expliquent leur démarche : ils découpent des lexèmes en morphèmes, sans toutefois motiver leur choix. C'est pourquoi nous nous basons sur Bonami et al. (2009: 112-125) qui s'interrogent sur le statut précis du suffixe -ion, en comparant plusieurs exemples, à savoir vexation, modification, dispersion, composition, pollution... Ces auteurs (Bonami et al., 2009: 106, 120-124) émettent l'hypothèse de l'existence d'un radical caché pour expliquer cette variation formelle. En comparant les noms en -io du latin avec les noms en -ion du français, ils constatent que ces derniers sont tous deux construits à partir d'un radical verbal particulier, qui se différencie des autres radicaux employés dans la flexion et qui peut souvent être irrégulier. Alors qu'en latin, ce radical spécial se réalise aussi bien dans la flexion que dans la dérivation, il ne se manifeste plus que dans les opérations constructionnelles en français et est donc un radical caché à la flexion. Ainsi, en latin, le verbe movere ("mouvoir") a trois radicaux : celui de l'infectum, /movē/, celui du perfectum, /mōv/, et un troisième radical, /mōt/. Dans la flexion, ce troisième radical est entre autres employé pour former le participe passé passif motus, mais aussi pour former des dérivés tels que motor ("moteur") et motio ("mouvement"). En français, les verbes susceptibles de former un radical caché peuvent l'emprunter directement au latin, en utilisant le "troisième radical", c'est-à-dire celui du supin, qui est idiosyncratique ; pensons par exemple à abstraction, dérivé d'abstraire sur la base de ce troisième radical. Si le radical caché n'est pas emprunté au latin, l'on forme un radical supplémentaire en /at/, puisqu'il est « le mode de formation par défaut du radical caché » (Bonami et al., 2009 : 124). Ainsi, le verbe vexer, qui utilise toujours le radical vexdans la flexion, forme son dérivé vexation à l'aide d'un radical caché en /at/ (Bonami et al., 2009 : 122). Les suffixes, et par extension, les affixes en général, correspondent à la plus petite partie du lexème que celui-ci a en commun avec d'autres lexèmes, comme le suffixe -ion dans composition, pollution, inclusion, réduction, location, réaction, rébellion. À l'instar de Bonami et al. (2009), nous décomposons donc desudation comme de-sudation, sudat- étant la base cachée (radical caché) de desudation, sud- sa racine. Bien entendu, les néologismes attestés dans notre corpus comportent d'autres suffixes que -ion, -eur/-rice et -if, comme par exemple les termes sudoral et sudorable, qui font partie de la même famille morphologique que desudation et resudation, puisqu'ils sont construits sur la même racine sud-. Ainsi, nous considérons sudora- comme la base de sudorable et de sudoral, leur suffixe correspondant alors à -ble dans le cas de sudorable et à -l dans le cas de sudoral. En effet, dans le premier cas, -ble est le suffixe, puisqu'il correspond au plus petit élément partagé par les termes sudorable, abominable, decourable, defaillable, faillable, grevable, putrefactible, putrescible et voluble, qui ont tous été relevés dans le corpus. Dans le deuxième cas, $-l$ est la plus petite partie du lexème que les mots sudoral, formical, 
putrimental, sincopal, pestilential, pestilencie $\underline{\underline{l}}$ et continue $\underline{\underline{l}}$ ont en commun, $-\mathrm{l}$ étant donc le suffixe, Les termes flegmatique, flegmasie et leucoflemantie quant à eux, sont tous les trois construits à partir des bases cachées de la base flegm-, respectivement flegmat-, flegmas-flemant-, et des suffixes -ique et -ie. ${ }^{10}$

Une série d'autres mises au point sont également nécessaires, dont la première concerne le statut d'emprunt vis-à-vis de celui de dérivé. Ainsi, sudoral pourrait être analysé comme le résultat d'une dérivation de la racine $s u d$-, empruntée au latin et formant la base cachée sudora-, et du suffixe -l. Néanmoins, le dérivé sudoralis est attesté en latin médiéval et il est donc plus probable que sudoral soit un emprunt, vu la situation linguistique au Moyen Âge. En d'autres termes, nous considérons toujours comme des emprunts les lexèmes français qui, en synchronie, pourraient être analysés comme des dérivés français à partir d'une racine/base empruntée au latin, si le dérivé lui-même est déjà attesté en latin. Il est effectivement impossible à prouver, selon les morphologues, que ces termes soient des dérivés ou des emprunts d'un dérivé latin (Goyens, 2013 : 49, Goyens \& Van Tricht, 2015 : 393, Goyens \& Szecel, 2017 : 156). En revanche, les termes possédant une racine/base latine, mais ne remontant pas à un étymon latin attesté, sont catégorisés comme des dérivés à partir d'une racine/base latine. Ainsi, sudorable est analysé comme un dérivé à partir de la racine latine sud-, parce qu'il n'y a aucune trace du terme latin sudorabilis dans les dictionnaires latins consultés.

Dans le cas des emprunts, l'on peut se demander s'il est légitime de les décomposer, alors qu'ils auraient été empruntés intégralement à une langue étrangère, en l'occurrence, au latin. Même si cela reste intéressant du point de vue étymologique, nous souhaitons avant tout réaliser une analyse synchronique de ces termes, qui ont par ailleurs été intégrés au français médiéval, ce dont témoigne l'adaptation de leurs finales aux normes phonétiques du français de l'époque (Goyens et al., 2017 : 394-395). Comme Bonami et al. (2009) l'ont montré pour le français moderne, il est possible de réanalyser des emprunts de termes latins en -io comme des dérivés construits à l'aide du suffixe -ion, ce dernier étant productif. Même si le traducteur médiéval recourt à un emprunt et crée ainsi un néologisme, celui-ci peut rapidement passer dans la langue, les lecteurs du texte ne se rendant plus compte du statut néologique qu'avait le terme à l'origine et pouvant le réanalyser, non plus comme un emprunt, mais comme un dérivé. Dans l'analyse qui suit, nous décomposons donc chaque terme en base et affixes, qu'il s'agisse d'un emprunt ou non.

Signalons enfin que nous n'analysons pas les liens entre les différents termes appartenant à une famille morphologique, c'est-à-dire que nous ne cherchons pas à identifier le terme qui serait à l'origine d'un autre terme, que ce soit un dérivé ou un composé, parce que cela pose un certain nombre de problèmes. Il serait par exemple possible de faire remonter le terme flegmasie à la forme flegme, dont celui-ci aurait été dérivé grâce au suffixe -ie, qui entraîne la formation de la base cachée flegmas-. Que faire alors du lexème apoplexie, qui ne semble pas être dérivé d'un autre mot, le terme simple *apoplexe n'existant pas en français médiéval, ni en français moderne d'ailleurs, mais où l'on reconnaît clairement le même suffixe -ie? Comment savoir si un verbe est la conversion ou le dérivé d'un substantif ou si le processus inverse s'est produit, c'est-à-dire si le substantif provient du verbe ? $^{11}$

Comme base théorique pour nos observations, nous faisons appel à la Morphologie des Constructions (Booij, 2010), qui est une application des principes de la Grammaire de Construction (cf. Goldberg, 1995, Croft, 2001, Hilpert, 2013, Traugott \& Trousdale, 2013, etc.) à la morphologie lexicale. Selon ces principes, la grammaire et le lexique se situent sur un continuum et la morphologie est « un ensemble structuré de constructions (paires formesens) au niveau du mot » (Booij, 2008 : 59), ce qui signifie que les lexèmes complexes sont des constructions au niveau du mot.

Le lexique y est conçu comme un lexique hiérarchisé comprenant différents niveaux d'abstraction, dont chaque niveau hérite les caractéristiques du niveau plus élevé. Ainsi, le 
composé hoofdingang «litt. tête-entrée ; entrée principale », qui est l'instanciation de schémas plus généraux, hérite de toutes leurs propriétés. Le schéma $[X Y]_{Y}$ montre que la tête sémantique et formelle $\mathrm{Y}$ des composés se situe toujours à droite en néerlandais. Le niveau plus concret, $[\mathrm{XN}]_{\mathrm{N}}$, montre que le terme hoofdingang fait partie des composés dont l'élément recteur $\mathrm{Y}$ est un nom $\mathrm{N}$, le $\mathrm{N}$ souscrit indiquant que le mot complet est également un nom. Le niveau suivant, $[\mathrm{NN}]_{\mathrm{N}}$, indique le constituant de gauche, à savoir hoofd, est également un nom dans le lexème hoofdingang. Toutefois, les niveaux plus concrets peuvent présenter des exceptions et être plus spécifiques sur le plan sémantique, sans devoir modifier le niveau le plus abstrait. Concrètement, le nom hoofd prend le sens de 'principal' lorsqu'il se combine avec un autre nom au sein d'un composé alors qu'il signifie 'tête' s'il est employé seul. La relation entre les constituants doit donc être déterminée pour chaque composé en fonction du sens de ses constituants et grâce aux connaissances encyclopédiques et contextuelles des locuteurs. L'arbre d'héritage multiple suivant représente la structure des composés dans le lexique du néerlandais en général, mais aussi les propriétés sémantiques particulières des composés du type $\left[[h o o f d]_{\mathrm{N}}[\mathrm{x}]_{\mathrm{N}}\right]_{\mathrm{N}}$ et de son instanciation hoofdingang, sans oublier les niveaux intermédiaires.

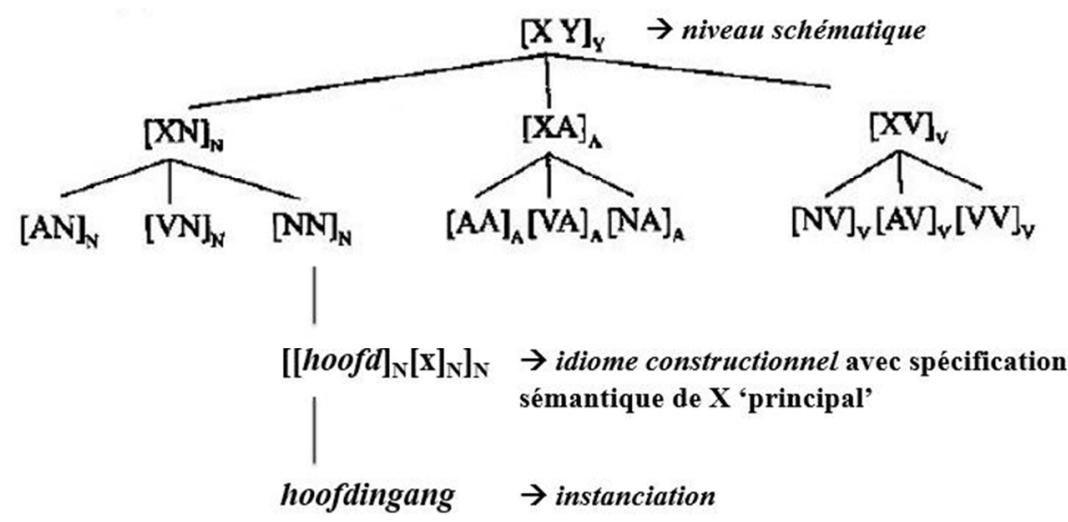

Fig. 1. Arbre d'héritage multiple pour la composition en néerlandais (Booij, 2008 : 54) et pour le composé hoofdingang en particulier, réalisé à partir des analyses de Booij (2008 : 59-60).

L'avantage fondamental de cette approche est que des généralisations sur des sous-ensembles de mots complexes partageant certaines propriétés sont possibles, mais que les différences entre ces mots complexes sont également représentées, sans perdre de vue ce qui est commun à l'ensemble. Les affixes, quant à eux, ne sont pas des entités indépendantes, mais leurs propriétés sont seulement accessibles grâce aux constructions dans lesquelles ils apparaissent.

En outre, au sein du lexique mental du locuteur, les mots concrets et les schémas de construction plus abstraits coexistent et permettent au locuteur de procéder directement à une dérivation complexe, sans avoir recours à un mot possible inexistant. Ainsi, le terme complexe on-beschrijf-elijk "in-descript-ible» en néerlandais, peut être immédiatement dérivé à partir du radical verbal beschrijf « décris » sans étape intermédiaire, à savoir le mot possible mais inexistant *beschrijfelijk «descriptible». Les schémas constructionnels $[o n-\mathrm{A}]_{\mathrm{A}}+[\mathrm{V} \text {-elijk }]_{\mathrm{A}}$ peuvent donc être unifiés en un seul schéma plus complexe, à savoir $\left[o n\left[[\mathrm{~V}-e l i j k]_{\mathrm{A}}\right]_{\mathrm{A}}\right.$. Si le locuteur est capable de former des lexèmes complexes de cette manière, il est tout à fait concevable qu'il construise des dérivés et des composés directement à partir d'une base/racine et d'un ou de plusieurs affixes, sans devoir les dériver d'autres lexèmes. Par ailleurs, les termes concrets sont présents dans le lexique mental du locuteur et correspondent à des schémas constructionnels plus abstraits. Le lexique mental du locuteur est dès lors un lexique dynamique, qui lui permet aussi de créer des néologismes en employant ces schémas de construction. 
Nous considérons dès lors la dérivation et la composition comme se faisant à partir des bases/racines des termes concernés et non d'autres mots complets. Ainsi, le lexème aplopexie est analysé comme un dérivé de la racine apoplex-, qui est empruntée au latin (RL), à l'aide du suffixe -ie.

Afin de vérifier notre hypothèse de recherche, nous déterminons pour chacune des neuf familles morphologiques étudiées combien de néologismes ont été forgés au cours du Moyen Âge et lesquels d'entre eux ont survécu en français après cette période, en conservant ou non leur sens médical. ${ }^{12}$ Ensuite, nous examinons si le lien formel avec le latin est solide ou plutôt faible et si la famille est déjà bien établie en moyen français, c'està-dire composée de plusieurs lexèmes. Enfin, nous étudions la présence de corrélations transparentes sur le plan formel et sémantique entre les lexèmes de cette famille, en vérifiant si la forme de leur racine renvoie toujours au même sens et que la forme des affixes employés ait systématiquement la même signification. Nous analysons ici trois familles morphologiques de façon détaillée avant de résumer les analyses des autres familles et de passer aux résultats généraux.

\subsection{Famille morphologique de cacochie}

La figure 2 représente la famille morphologique des cinq termes médicaux de la famille de cacochie, à savoir cacochie, cacochime, cacochimie, cacochimeux et cacochimique. Seuls cacochymie et cacochyme sont encore utilisés en français, bien que ce dernier soit considéré comme « vieux » et «plaisant » par le TLFi et qu'il ne garde pas de signification médicale. Même si cacochymie est encore employé en médecine, le TLFi le qualifie de « rare ». Tous ces mots sont des emprunts au latin, cacochie au latin médiéval cacochiam ${ }^{13}$ " maladie, affection », cacochime et cacochimie respectivement au bas latin cacochymum «qui est doté d'un mauvais suc, d'où : de constitution appauvrie (en parlant du corps humain)» et cacochymiam «mauvais état des humeurs », excepté cacochimeux et cacochimique, qui sont tous deux dérivés de la racine latine (TLFi, DLD). Le lien formel entre ces lexèmes et leur étymon latin est donc solide.

Les relations formelles et sémantiques entre les néologismes de cette famille morphologique sont par ailleurs systématiques, vu l'utilisation des suffixes -ie, désignant un " état (pathologique)», -eux, «qui produit la substance désignée par la base » et -ique, « qui est propre à » (TLFi). La racine cacoch- renforce également les corrélations formelles et sémantiques de la famille, parce qu'elle conserve la même forme et signifie toujours «mauvaise humeur ». 

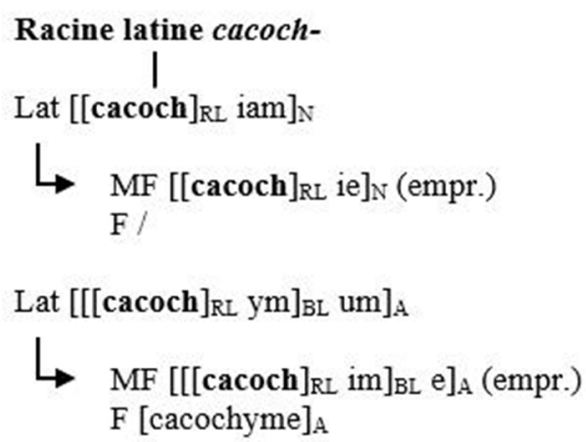

Lat $\left[\left[[\mathbf{c a c o c h}]_{\mathrm{RL}} \mathrm{ym}\right]_{\mathrm{BL}} \text { iam }\right]_{\mathrm{N}}$

$\longrightarrow$ MF $\left[\left[[\mathbf{c a c o c h}]_{R L} \text { im }\right]_{B L} \text { ie }\right]_{N}($ empr.) $\mathrm{F}[\text { cacochymie }]_{\mathrm{N}}$

Lat $\left[[\operatorname{cacoch}]_{\mathrm{RL}} \mathrm{ym}\right]_{\mathrm{BL}}$

$\longrightarrow$ MF $\left[\left[[\mathbf{c a c o c h}]_{\mathrm{RL}} \text { im }\right]_{\mathrm{BL}} \text { ique }\right]_{\mathrm{A}}$ (dér. lat.) $\mathrm{F} /$

Lat $\left[[\operatorname{cacoch}]_{\mathrm{RL}} \mathrm{ym}\right]_{\mathrm{BL}}$

$\longrightarrow \mathrm{MF}\left[\left[[\mathbf{c a c o c h}]_{\mathrm{RL}} \text { im }\right]_{\mathrm{BL}} \text { eux }\right]_{\mathrm{A}}$ (dér. lat.) $\mathrm{F} /$

Fig. 2. Arbre morphologique construit à partir de la racine latine cacoch- ${ }^{14}$

Ce schéma peut donc se lire de la façon suivante : cacochimique et cacochimeux sont tous les deux des dérivés construits à partir de la base latine cacochym-, mais ne remontent donc pas à un terme latin en tant que tel, comme le sont les emprunts.

\subsection{Famille morphologique de chancre}

Une deuxième famille, partageant la même racine héréditaire chancr-, est formée par chancre (DMF) et ses dérivés chancreux, enchancrir et achancrir, soit quatre lexèmes. Il s'agit dès lors d'une famille morphologique bien établie, dont le terme héréditaire chancre et le néologisme chancreux sont les seuls à avoir survécu en français, conservant tous deux un sens médical, bien que chancreux soit plus fréquemment employé dans le domaine de la sylviculture (TLFi). Vu qu'il s'agit d'une forme héréditaire et de ses dérivés, le lien formel avec le latin est faible.

En moyen français, la signification des termes médicaux de la famille morphologique est compositionnelle : si l'on comprend la racine chancr-, l'on peut facilement déduire le sens des mots concernés à l'aide de leur affixe, le suffixe -eux dans chancreux désignant " qui est de la substance désignée par la base, qui produit la substance désignée par la base » ou «qui indique la présence de la base » (TLFi). Chancreux a donc le sens d' "atteint de chancre ; qui est de la nature du chancre ». Le préfixe en-, quant à lui, « traduit l'entrée dans un état, l'acquisition d'une forme ou d'une qualité nouvelle» (TLFi), ce qui permet de comprendre enchancrir, signifiant «se développer en chancre, devenir chancreux ». Les deux verbes enchancrir et achancrir ont été dérivés de la racine chancr- à l'aide de la désinence verbale -ir, à moins qu'ils ne soient le résultat de la conversion de chancre en verbe ainsi que d'une préfixation à l'aide du préfixe en-, dans le premier cas, et du préfixe $a-$, dans le deuxième. Étant donné que nous considérons la dérivation et la composition 
comme réalisées à partir des bases/racines des lexèmes concernés et non d'autres termes complets, nous optons pour la première possibilité. Le terme achancrir est par ailleurs un synonyme d'enchancrir ${ }^{15}$, malgré la présence du préfixe $a$-, qui "exprime une idée de passage d'un état à un autre » (TLFi). La famille morphologique n'est donc pas transparente en ce qui concerne l'emploi du préfixe $a$ - et en-, qui semblent recevoir la même signification. Néanmoins, la racine chancr- reste stable, aussi bien sur le plan formel (chancr-) que sémantique ( chancre »).

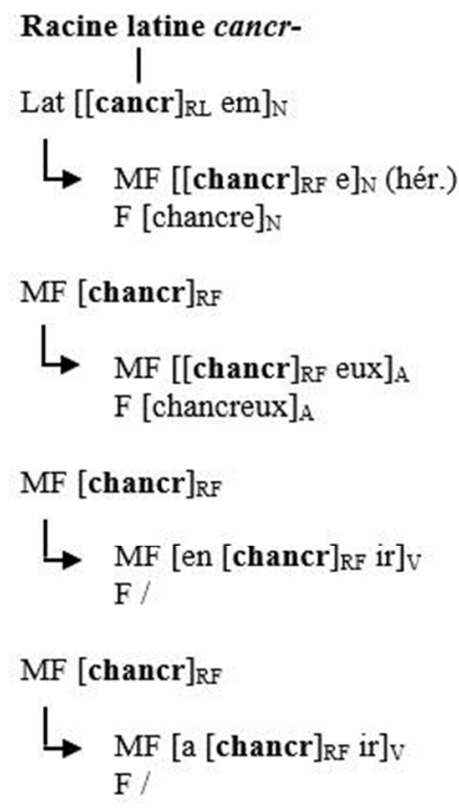

Fig. 3. Arbre morphologique construit à partir de la racine latine cancr-évoluant à chancr-. ${ }^{16}$

\subsection{Famille morphologique de flegme}

La troisième famille morphologique analysée est constituée des quatre néologismes flegme, flegmatique, flegmasie et leucoflemantie. ${ }^{17}$ Tous ces termes, hormis leucoflemantie, survivent en français avec un sens médical, mais sont caractérisés de vieillis par le TLFi. Flegme et flegmatique s'emploient actuellement surtout pour désigner respectivement le «caractère d'une personne calme et imperturbable, qui garde son sang-froid en toutes circonstances » et l'adjectif «calme et imperturbable, qui garde son sang-froid ». Flegme, flegmatique et flegmasie ont tous été empruntés au latin, à savoir respectivement à flegmam "humeur» en bas latin, à flegmaticum "qui abonde en flegme » et à flegmasiam « inflammation » en latin médiéval, et ont donc un lien formel solide avec le latin (TLFi, ALD). Leucoflemantie, quant à lui, est un composé, construit à partir de la variante de la racine latine flem- ${ }^{18}$

Les corrélations entre les membres de cette famille morphologique sont transparentes, vu l'utilisation des suffixes -ie, désignant un « état (pathologique) » et -ique, " relatif à, qui est propre à ", qui permettent de comprendre flegmatique et flegmasie, si l'on connaît le sens de flegme (TLFi). Le composé leucoflemantie "sorte d'hydropisie ", quant à lui, est également transparent, le suffixe -ie conservant le sens d' 'état (pathologique)» et l'élément leuco- semblant être motivé. En effet, la leucoflemantie est une "sorte d'hydropisie provoquée par le flegme blanc », leuco- renvoyant alors au fait que le flegme provoquant cette maladie est de couleur blanche. Par ailleurs, la racine flegm- reste stable formellement et sémantiquement dans toute la famille (« flegme »). 


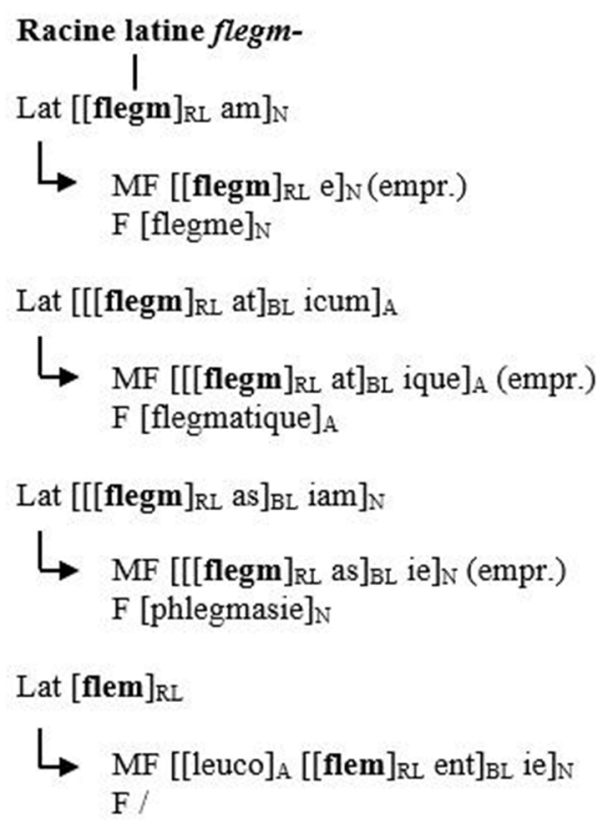

Fig. 4. Arbre morphologique construit à partir de la racine latine flegm- ${ }^{19}$

\subsection{Familles morphologiques de frenesie, apoplexie, apostume, asme, sudoral et sueur}

Les six autres familles morphologiques, à savoir (4) dissinterie, dissinterique; (5) apoplexie, apoplectique; (6) apostume, apostumation, apostumeux, apostumer ; (7) asme, asmatique; (8) sudoral, sudorable, resudation, desudation; et enfin, (9) suer, sueur ${ }^{20}$ ont été analysées selon la même méthode.

Il est ressorti de ces analyses que le nombre de néologismes formés au cours de l'époque médiévale peut s'élever de deux à quatre. Précisons toutefois que la neuvième famille ne contient aucun néologisme, les deux termes qui la composent étant des formes héréditaires. Tous les néologismes survivent en français moderne en conservant un sens médical, excepté apostumation, apostumeux qui font partie de la sixième famille et, sudorable, desudation, resudation, qui appartiennent à la huitième famille.

Le lien formel entre tous les néologismes et le latin est solide, tous ces termes étant des emprunts au latin ou des dérivés à partir de racines latines. Cependant, les formes héréditaires de la neuvième famille s'éloignent beaucoup du latin sur le plan formel.

Enfin, toutes ces familles - sauf la huitième - sont transparentes sur le plan formel et sémantique, la forme de leur racine conservant systématiquement la même signification et la forme des affixes employés renvoyant toujours au même sens. En effet, à l'intérieur des termes resudation "action de traverser un corps, en parlant de fluides corporels » et desudation "pustule provoquée par la sueur» de la huitième famille, la racine sud- ne renvoie plus à la sueur à proprement parler. Dans les formes sudoral et sudorable, toutefois, la racine $s u d$ - renvoie encore à la sueur. Sudoral est construit à l'aide du suffixe - $l$ « relatif à » et signifie donc « relatif à la sueur, provoqué par la sueur». En général, le suffixe -ble, quant à lui, a le sens de "qui cause, qui produit», alors que sudorable est défini comme "qui peut se transformer en sueur ». La signification de -ble se rapproche donc du sens qu'il adopte quand il est le « suffixe formateur d'adjectifs à partir de verbes transitifs directs et exprimant la possibilité passive ('que l'on peut' + infinitif) », comme par exemple dans absorbable «qui peut être absorbé » (TLFi). Dès lors, les nuances sémantiques différentes 
de la racine et du suffixe -ble compliquent une bonne compréhension de ces termes pour le locuteur.

\section{Résultats}

Selon notre hypothèse de départ, la survie des néologismes médicaux dépend de leur relation formelle avec l'élément latin dont ils sont issus : plus cette relation est forte et plus le néologisme fait partie intégrante d'une famille morphologique bien établie et transparente en ce qui concerne la forme et le sens de ses membres, mieux le néologisme se maintiendra, alors que les créations françaises indigènes disparaîtront plus facilement, en tout cas dans un emploi médical.

L'analyse des neuf familles morphologiques (voir 2) ne confirme pas cette hypothèse dans son intégralité, puisque la deuxième condition n'est pas remplie. En effet, la survie d'un terme médical emprunté au latin ou dérivé du latin ne semble pas être influencée par le nombre de termes appartenant à sa famille morphologique. Ainsi, même si la famille morphologique d'apoplexie et d'apoplectique ne contient que deux termes, ces néologismes survivent en conservant leur signification médicale. Dans les familles morphologiques d'aposteme et de desudation, chacune composée de quatre membres, seuls deux termes médicaux parviennent à se maintenir après le moyen français.

En revanche, le critère de la transparence semble jouer un rôle significatif pour la survie des termes d'une famille morphologique. Deux des neuf familles morphologiques manquent de transparence en ce qui concerne leur racine ou l'emploi des affixes, à savoir celle de chancre (2) et de sudoral (8). Ce manque de transparence sémantique de la racine s'observe lorsque cette dernière conserve la même forme dans tous les lexèmes d'une famille morphologique, mais change de sens dans certains termes. Citons par exemple la famille de sudoral (8), qui présente une instabilité sémantique de la racine sud-, qui reste stable sur le plan formel : en effet, la racine sud-perd le sens de sueur dans les néologismes resudation " action de traverser un corps, en parlant de fluides corporels » et desudation " pustule provoquée par la sueur ». Au sein des autres termes de cette famille, à savoir sudoral et sudorable, la racine conserve la signification de sueur. Il est intéressant de constater qu'aussi bien les termes resudation que desudation ne sont plus utilisés en français, ce qui montre donc que le manque de transparence sémantique de la racine peut provoquer la disparition des termes concernés. En outre, dans cette même famille (8), le suffixe -ble, présent dans le lexème sudorable, n'a pas le sens habituel de « qui cause, qui produit », mais doit être interprété comme « qui peut se transformer en ».

Dans les synonymes achancrir et enchancrir, les deux préfixes $a$ - et en- ont le même sens et se font concurrence. Par conséquent, la transparence sémantique des affixes est donc importante pour la survie des termes des familles morphologiques, sudorable, achancrir et enchancrir ne se maintenant pas en français. Au total, le taux de survie des termes appartenant à ces familles morphologiques s'élève seulement à $38 \%$, les cinq lexèmes concernés disparaissant tous, alors que ce pourcentage est plus important dans les familles transparentes $(71 \%)$.

En résumé, la transparence sémantique de la racine et des affixes aide donc effectivement une terminologie à se renforcer et à se maintenir. La majorité des emprunts ou des dérivés latins gardent par ailleurs leur sens médical (13 termes médicaux contre un seul lexème employé en langage courant ${ }^{21}$ ) alors qu'une seule création française, à savoir le dérivé chancreux, qui fait partie de la famille morphologique de chancre, est encore employée actuellement avec un sens médical. En général, les emprunts et dérivés latins ont donc effectivement plus de chance de survivre, c'est-à-dire $71 \%$ (15 sur 21$)$, parce qu'ils sont formellement beaucoup plus proches de leur étymon latin que les dérivés français. Sur sept familles morphologiques composées d'emprunts ou de dérivés latins (toutes les familles, excepté les familles 2 et 9), le nombre de lexèmes de seulement quatre familles, à 
savoir les familles 1, 3, 6 et 8, diminue après le moyen français. Dans les deux familles morphologiques qui contiennent des formes héréditaires et des créations françaises, ces dernières disparaissent toutes, sauf le dérivé chancreux, déjà mentionné ci-dessus, le taux de survie de ces termes s'élevant donc à 33\% (1 sur 3), c'est-à-dire un peu moins de la moitié de celui des termes des familles composées d'emprunts ou de dérivés latins (61\%).

Néanmoins, ces résultats sont provisoires, vu qu'un plus grand nombre d'exemples devra encore être analysé afin de pouvoir présenter des conclusions générales applicables à toutes les familles morphologiques sélectionnées dans notre corpus. Toutefois, nous pensons que ces tendances se confirmeront lorsque notre étude, qui est en cours d'élaboration, sera terminée.

\section{Application de la Morphologie des Constructions}

Dans une étape ultérieure, les termes ont été classés par affixe et réanalysés selon la Morphologie des Constructions (Booij, 2010).

Concrètement, voici deux exemples d'arbres d'héritage multiple qui ont pu être créés à partir des données de notre corpus pour les termes construits à l'aide des suffixes -ie et -ique.

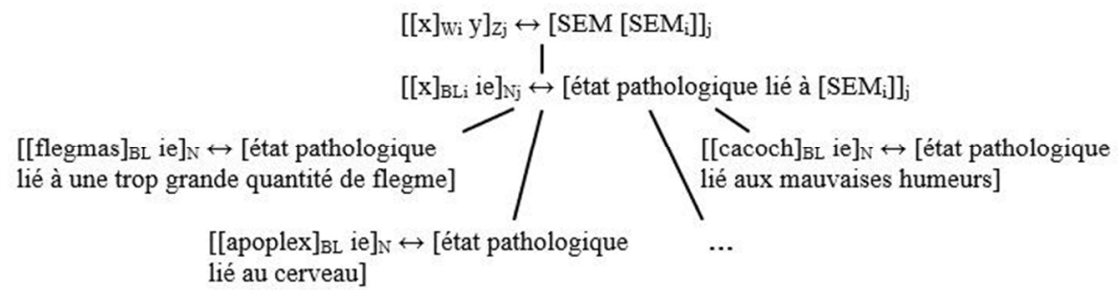

Fig. 5. Arbre d'héritage multiple des termes construits à l'aide du suffixe -ie.

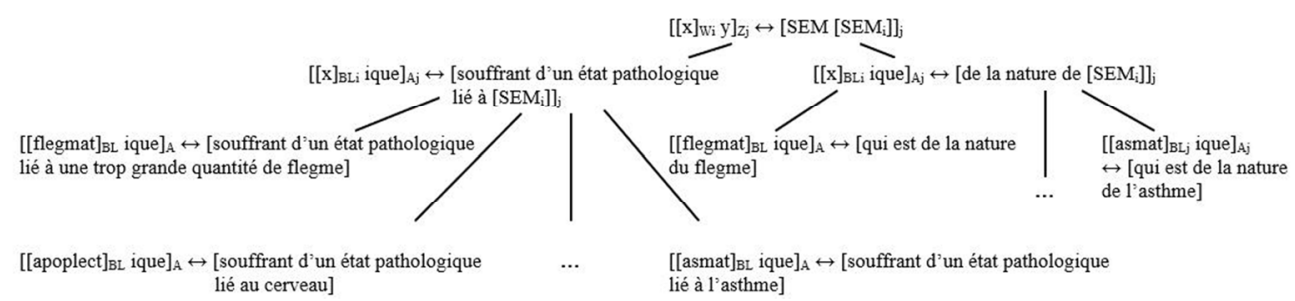

Fig. 6. Arbre d'héritage multiple des termes construits à l'aide du suffixe-ique.

Dans le schéma constructionnel $\left[[x]_{W_{i}} y\right]_{Z j} \leftrightarrow\left[\left[S_{\mathrm{SEM}}\right] \quad S E M\right]_{j}$, qui s'inspire de Booij (2010 : 17) et Masini \& Audring (Forth.), l'élément de gauche $X$ est la forme phonologique que peut adopter la base du terme et la variable $Y$, le suffixe, qui ne peut apparaître qu'en combinaison avec une base. La nature de la base $X$ est indiquée à l'aide de la majuscule souscrite $W$, qui est une base latine (BL) dans ce cas-ci, mais pourrait également être une base française dans d'autres cas. La catégorie lexicale principale à laquelle appartient le mot dans son intégralité est par ailleurs représentée par la majuscule souscrite $Z$. La flèche récursive symbolise la correspondance systématique entre la forme et sa signification. $\mathrm{Vu}$ que toute construction est composée d'une paire de forme et de sens, la signification de la construction dans son intégralité est spécifiée grâce à la minuscule souscrite $j$, même si la nature de la relation sémantique doit être déterminée pour chaque composé individuel. Par ailleurs, le sens du composé est compositionnel, $\left[\mathrm{SEM}_{\mathrm{i}}\right]$ renvoyant à la signification de la base $X$ et SEM, à celle du suffixe $Y$. Lorsque l'on combine ces deux sens, l'on peut accéder à la signification $j$ du terme dans son intégralité.

Ces deux arbres d'héritage multiple nous permettent donc de visualiser les propriétés formelles et sémantiques des termes construits en français médiéval à partir des suffixe -ie 
et -ique, qui en combinaison avec des bases latines forment le substantif désignant « un état pathologique lié à $\mathrm{SEM}_{\mathrm{i}}$ » et l'adjectif, « souffrant d'un état pathologique lié à $\mathrm{SEM}_{\mathrm{i}}$ » ou «de la nature de $\mathrm{SEM}_{\mathrm{j}}$ », respectivement. Alors que les adjectifs en -ique peuvent avoir deux sens, le niveau schématique reste inchangé, ce qui montre que les niveaux plus bas héritent des propriétés formelles et sémantiques des niveaux plus abstraits et que des caractéristiques individuelles peuvent se manifester aux niveaux plus concrets.

Bien entendu, il faudra analyser dans notre corpus tous les termes construits à l'aide des suffixes -ie et -ique afin de représenter adéquatement la richesse sémantique de ces lexèmes. Les mots formés à partir d'autres affixes feront également l'objet d'une telle réanalyse grâce à l'application de la Morphologie des Constructions, permettant de former un vaste réseau morphologique hiérarchique, où les liens entre les lexèmes affixés seront visualisés et clarifiés.

\section{Références bibliographiques}

ALD. Aristoteles Latinus Database, clt.brepolis.net/ald. Turnhout: Brepols Publishers.

Amiot, D. (2011). Paradigmes, radicaux supplétifs et constituants néoclassiques en morphologie constructionnelle. Dans F. Hrubaru \& E. Moline (éds.), Paradigmes en morphologie constructionnelle (pp. 21-36). Constanta: Echinox.

Apothéloz, D. (2002). La construction du lexique français. Principes de morphologie dérivationnelle. Paris: Ophrys.

Bonami, O., Boyé, G. \& Kerleroux, F. (2009). L'allomorphie radicale et la relation flexionconstruction. Dans B. Fradin, F. Kerleroux \& M. Plénat (éds.), Aperçus de morphologie du français (pp. Saint-Denis: Presses Universitaires de Vincennes.

Booij, G. (2008). Composition et morphologie des constructions. Dans D. Amiot (éd.), La composition dans une perspective typologique (pp. 49-73). Arras: Artois.

Booij, G. (2010). Construction Morphology. Oxford: Oxford University Press.

Bos, A. (Ed.). (1897-1898). La Chirurgie de maître Henri de Mondeville. Traduction contemporaine de l'auteur, publiée d'après le ms. unique de la Bibliothèque nationale (Vol. 2). Paris: Firmin Didot.

CHrOMed. (À par.). Historical Corpus of French Medical Texts: sous la direction de Michèle Goyens, en collaboration avec Céline Szecel et Ildiko Van Tricht.

Croft, W. (2001). Radical construction grammar. Oxford: Oxford University Press.

Dal, G. (Ed.). (2003). La productivité morphologique en questions et en expérimentations (Vol. 140). Paris: Larousse.

Deroy, L. (1956). L'emprunt linguistique. Paris: Belles Lettres.

DLD. Database of Latin Dictionaries, clt.brepolis.net/dld/. Turnhout: Brepols.

DMF2015. Dictionnaire du Moyen Français, www.atilf.fr/dmf. Nancy: ATILF CNRS Université de Lorraine.

Ducos, J. (1998). La météorologie en français au Moyen Âge (XIIIe - XIVe siècle). Paris: Champion.

Godefroy, F. (1880-1902). Dictionnaire de l'ancienne langue française et de tous ses dialectes du IXe au XVe siècle. Paris: F. Vieweg.

Goldberg, A. E. (1995). Constructions. A construction grammar approach to argument structure. Chicago: University of Chicago Press.

Goyens, M. (2013). Le sort des néologismes dans la langue des sciences au Moyen Âge : une question de morphologie? Neologica, 7, 41-56.

Goyens, M. \& Szecel, C. (2017). Autorité du latin et transparence constructionnelle : le sort des néologismes médiévaux dans le domaine médical. Diachroniques, 7, 141-165.

Goyens, M., Szecel, C. \& Van Goethem, K. (2017). Une famille qui fait 'suer' : problèmes d'analyse des néologismes médiévaux 'sudoral', 'sudorable', 'resudation' et 'desudation'. Dans S. Prévost \& B. Fagard (éds.), Le français en diachronie. Dépendances 
syntaxiques, morphosyntaxe verbale, grammaticalisation (pp. 371-403). Berne: Peter Lang.

Goyens, M. \& Van Tricht, I. (2015). Albathe face à pustule : disparition versus lexicalisation des néologismes en français medieval. Dans C. Badiou-Monferran \& T. Verjans (éds.), Disparitions. Contributions à l'étude du changement linguistique (pp. 389-405). Paris: Champion.

Hathout, N. (2011). Une approche topologique de la construction des mots : propositions théoriques et application à la préfixation en anti. Dans M. Roché, G. Boyé, N. Hathout, S. Lignon \& M. Plénat (éds.), Des Unités Morphologiques au Lexique (pp. 251-318). Paris: Hermès.

Hilpert, M. (2013). Constructional change in English. Developments in allomorphy, word formation, and syntax. Cambridge: Cambridge University Press.

Joly, G. (1995). Précis de phonétique historique du français. Paris: Armand Collin.

Lusignan, S. (1989). La topique de la translatio studii et les traductions françaises de textes savants au XIVe siècle. Dans G. Contamine (éd.), Traduction et traducteurs au Moyen Âge (pp. 303-315). Paris: Editions du CNRS.

Masini, F. \& Audring, J. (Forth.). Construction Morphology. Dans J. Audring \& F. Masini (éds.), The Oxford Handbook of Construction Morphology (pp. ---). Oxford: Oxford University Press.

Meunier, F. (2003). La notion de productivité morphologique: modèle psycholinguistiques et données expérimentales. Langue Française, 140, 24-37.

Namer, F. (2003). Productivité morphologique, représentativité et complexité de la base: le système MoQuête. Langue Française, 140, 79-101.

Städtler, T. (2007). Le traducteur, créateur de néologismes: le cas de Nicole Oresme. Dans O. Bertrand, H. Gerner \& B. Stumpf (éds.), Lexiques scientifiques et techniques. Constitution et approche historique (pp. 47-61). Palaiseau: Éditions de l'École Polytechnique.

Szecel, C. (2017). L'analyse de néologismes médicaux du Moyen Âge à l'aide du corpus ChROMED. Une discussion des notions base, racine, radical et thème. Papers of the $L S B, 11,1-14$.

Tobler, A. \& Lommatzsch, E. (1915). Altfranzösisches Wörterbuch. Adolf Toblers nachgelassene Materialien bearbeitet und herausgegeben von Ehrhard Lommatzsch (Vol. 11). Berlin/Wiesbaden: Fr. Steiner.

Traugott, E. \& Trousdale, G. (2013). Constructionalization and Constructional Changes. Oxford: Oxford University Press.

Tribout, D. (2010). Les conversions de nom à verbe et de verbe à nom en français. Paris: Université Paris Diderot (Paris 7).

Van Tricht, I. (2015). La science en texte et contexte: la terminologie médicale française utilisée dans les Problèmes d'Evrart de Conty à la lumière du discours médical médiéval. Leuven: KU Leuven.

Von Wartburg, W. (1922-). Französisches etymologisches Wörterbuch: eine Darstellung des galloromanischen Sprachschatzes. Basel: Zbinden.

Wermuth, C. \& Verplaetse, H. (Forth.). Medical terminology in the Western World : current situation. Dans Handbook of Terminology Vol. 2 (pp. ---). Amsterdam: Benjamins.

\footnotetext{
${ }^{1}$ La notion de maladie est à comprendre au sens médiéval du terme, la médecine du Moyen Âge étant basée sur la théorie des quatre humeurs.

${ }^{2}$ Nous avons consulté le FEW (Von Wartburg, 1922-), qui est un dictionnaire étymologique ainsi que le Tobler et Lommatzsch (Tobler \& Lommatzsch, 1915), qui est un dictionnaire de l'ancien français. D'autres dictionnaires du moyen français, comme le Godefroy (Godefroy, 1880-1902) et le DMF
} 
2015 ont aussi été employés. Le TLFi, quant à lui, donne également des informations sur la première attestation du terme concerné, et sur son origine et étymologie. Pour identifier les étymons latins des emprunts, nous avons fait appel à la DLD, qui est une base de données de dictionnaires de latin classique, bas latin et latin médiéval et à l'ALD, une banque de données contenant des traductions médiévales latines d'Aristote.

${ }^{3}$ Ainsi, les formes fleugme et achancrer ont par exemple été assimilées à flegme et à achancrir, et la forme dialectale cranche typique de la Picardie et de la Wallonie (DMF), à chancre.

${ }_{5}^{4}$ À savoir, « (personne) qui est d'une santé fragile » et «état de fragilité dû à la vieillesse ».

${ }^{5}$ Pourtant, le néologisme cacochie aurait aussi pu se maintenir avec un sens différent, à savoir "gonflement des membres », sans expliciter la cause de ce gonflement, les mauvaises humeurs.

${ }^{6}$ La présente étude a été réalisée dans le cadre du projet Latin authority and constructional transparency: neologisms in the French medical vocabulary of the Middle Ages and their fate, subventionné par le Fonds de la recherche de la KU Leuven (OT/14/047). Il est dirigé par le professeur Goyens (KU Leuven) et codirigé par le professeur Van Goethem (F.R.S.-FNRS \& Université catholique de Louvain).Voir aussi Goyens \& Szecel (2017) et Goyens et al. (2017).

${ }^{7}$ Pour un aperçu de la littérature à propos des notions problématiques comme base, racine, radical et thème, nous renvoyons à Szecel (2017).

${ }^{8}$ Nous avons fait le choix de ne pas compléter ces familles à l'aide des dictionnaires, mais de nous limiter à celles attestées dans notre corpus.

${ }^{9}$ Voici la signification de ces termes qui ont été définis à partir du corpus : (1) cacochie « gonflement des membres, provoqué par de mauvaises humeurs (le malade a le visage pâle et jaunâtre, les yeux et les pieds gonflés, parfois, les mains) », cacochime « atteint de cacochie, plein de mauvaises humeurs ; hydropique ; personne souffrant de cacochie », cacochimeux « qui engendre de mauvaises humeurs ", cacochimie "corruption des humeurs, pouvant entraîner la cacochie », cacochimique "[humeur] cacochymique : mauvaise [humeur] »; (2) chancre "ulcération qui a tendance à s'étendre, chancre », chancreux «atteint de chancre; qui est de la nature du chancre», enchancrir «se développer en chancre, devenir chancreux », achancrir « se développer en chancre, devenir chancreux »; (3) flegme «flegme", flegmatique "qui est propre au flegme; qui abonde en flegme; (personne) qui a beaucoup de flegme ", flegmasie " inflammation interne », leucoflemantie "sorte d'hydropisie »; (4) dissinterie «flux des humeurs, désordre intestinal accompagné de diarrhée », dissinterique "[flux] intestinal, diarrhée; flux des humeurs »; (5) apoplexie «maladie du cerveau provoquant la perte de la connaissance et la paralysie », apoplectique "(personne) souffrant d'apoplexie »; (6) apostume "tumeur, abcès", apostumation "fait de se former en apostume, formation d'un apostume", apostumeux «constitutif d'un apostume ; affecté par un apostume», apostumer «transformer en apostume»; (7) asme "maladie provoquant des difficultés respiratoires », asmatique "[maladie, passion] asmatique : asthme ; (personne) souffrant d'asthme; relatif à l'asthme »; (8) sudoral "relatif à la sueur, provoqué par la sueur», sudorable "qui peut se transformer en sueur», resudation "action de traverser un corps, en parlant de fluides corporels», desudation "pustule provoquée par la sueur»; (9) suer « suer », sueur « sueur».

${ }^{10}$ Voir aussi Szecel (2017) pour la décomposition des néologismes en base, racine et affixe(s).

${ }^{11}$ Nous ne pouvons malheureusement pas entrer dans les détails du concept de la conversion, mais pour plus d'information, nous renvoyons à Kerleroux (2009) et pour une analyse poussée de ce procédé, à Tribout (2010).

${ }^{12}$ Concrètement, nous vérifions si la forme concernée figure encore dans le TLFi. Même si certains termes y sont qualifiés de vieillis, ils ont quand même existé jusqu'au $19^{\mathrm{e}}$ ou $20^{\mathrm{e}}$ siècle, bien qu'ils soient désuets aujourd'hui. On considère en général le français moderne comme la langue parlée à partir de la Révolution française (1789) jusqu'à aujourd'hui. Pour nous, le terme survit donc s'il figure dans le TLFi.

${ }^{13}$ En général, les termes héréditaires remontent à une forme latine à l'accusatif (Joly, 1995). Dès lors, nous considérons les emprunts comme provenant de leur étymon à la forme accusative (Goyens et al., 2017 : 395) et cacochie serait alors issu de cacochiam en latin.

${ }^{14}$ Dans ce schéma, l'abréviation $L a t$ renvoie à 'latin', $M F$, à 'moyen français' et $F$, à 'français' devant les crochets. La lettre souscrite $N$ signifie 'nom' et $A$, 'adjectif'. $B L$ désigne 'base latine' et $R L$, 'racine latine'. L'abréviation empr. doit être comprise comme 'emprunt intégré' et dér. lat., comme 'dérivé à partir d'une racine latine. Les affixes ne sont pas indiqués par une abréviation.

${ }^{15}$ Les deux passages suivants du corpus illustrent la synonymie d'achancrir et enchancrir : 
«Aussi est ce mauvaise chose par voye de cause, pour ce telle colle noire et aduste passant par les membres dedens ulcere et escorche et fait achancrir, mez telle malice n'est pas es autres especes de cole noire ou de melancolie non naturelle qui est tout un. " Martin de Saint-Gilles, Le Livre des amphorismes Ypocras, 1363 (manuscrit Paris, BnF, fr. 24246 : f. 84ra, transcription de Van Tricht).

«Espoir cele mamele commençoit a enchancrir, ou diversion fu faite du flus de l'umeur a icele du lieu de la mamele coupee (et ceste desraine cause me semble meilleur). » Henri de Mondeville, Chirurgie, 1314 (Bos, 1897-1898 : tome 2, §2093).

${ }^{16}$ Pour les abréviations déjà utilisées, nous renvoyons le lecteur à la note 12. Dans l'arbre de la troisième figure, la lettre souscrite $V$ désigne 'verbe' et $R F$, 'racine française'. L'abréviation hér., quant à elle, signifie 'forme héréditaire'.

${ }^{17}$ Cette famille a également été analysée dans Goyens \& Szecel (2017: 149-155).

${ }^{18}$ Même si le terme leucoflegmantia figure dans la DLD sous le lemme leucophlegmatia, nous ne trouvons pas la forme leucoflemantia. C'est pourquoi nous ne considérons pas leucoflemantie comme un emprunt, mais plutôt comme un dérivé à partir de la variante flem-, attestée en latin médiéval, de la racine latine flegm-.

${ }^{19}$ Les abréviations employées dans cet arbre sont les mêmes que celles qui figurent dans la note 12.

${ }^{20} \mathrm{La}$ huitième et neuvième familles ont été étudiées en détail dans Goyens et al. (2017 : 378-392).

${ }^{21}$ Les 13 emprunts ou dérivés latins qui conservent leur sens médical en français sont cacochimie, flegme, flegmatique, flegmasie, dissinterie, dissinterique, apoplexie, apoplectique, apostume, apostumer, asme, asmatique et sudoral. Nous renvoyons au TLFi pour la signification de tous ces termes. Le lexème cacochime, quant à lui, n'est plus médical, mais est employé en langage courant, où il reçoit un sens plus figuré, à savoir « (personne) qui est d'une santé fragile » (TLFi). 